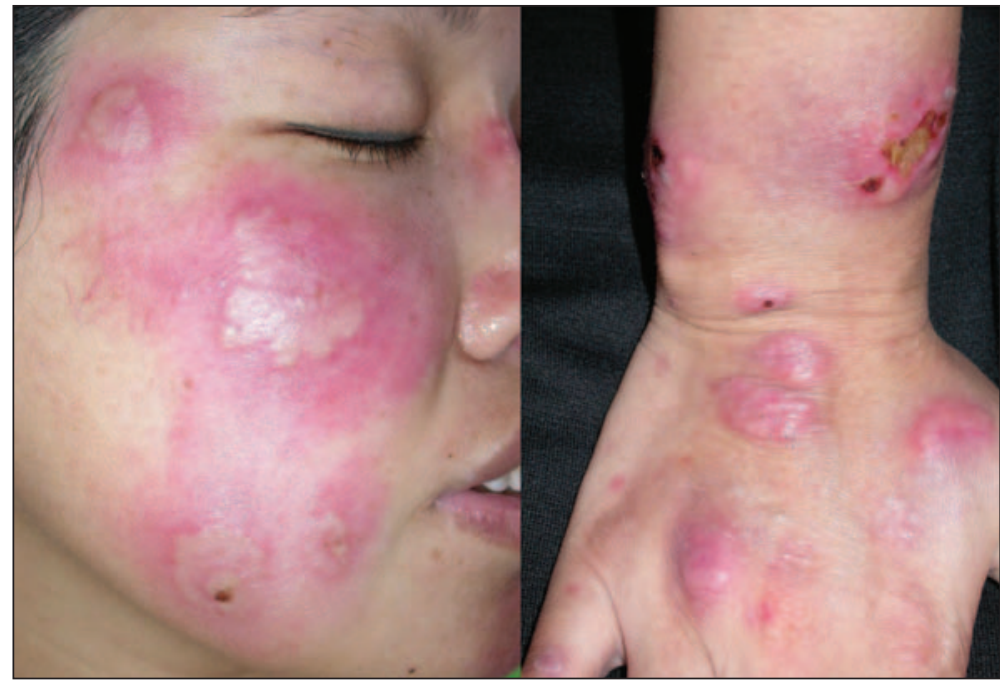

Figure 1: The patient had infiltrated and well circumscribed, purple-red plaques with a pustular component on her face (left) and right hand (right).

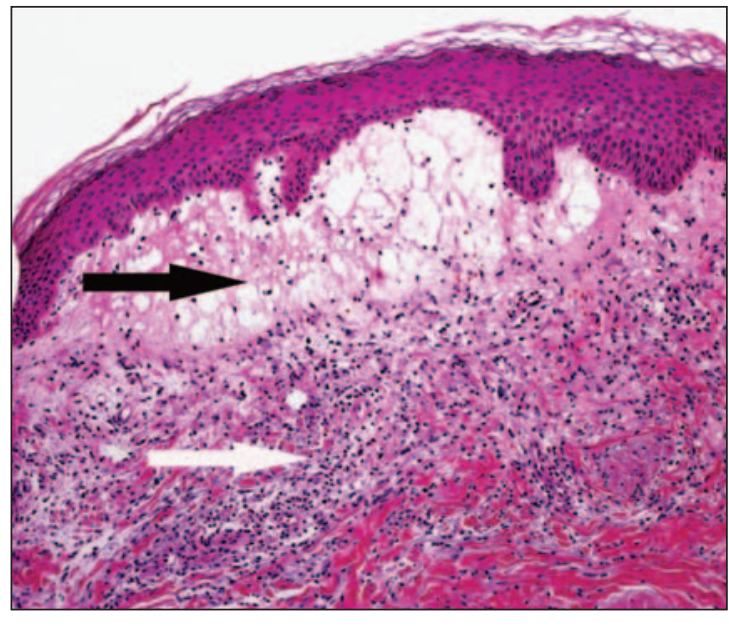

Figure 2: Pronounced edema in the papillary dermis (black arrow) with diffuse neutrophilic infiltration in the upper and mid dermis (white arrow), with no leukocytoclastic vasculitis.

\title{
Sweet syndrome during pregnancy
}

\section{A} 29-year-old woman in the 20th week of her second pregnancy presented with a fever and a 2-week history of painful, pruitic skin lesions that had appeared suddenly on her neck and face and had spread to her trunk and limbs (Figure 1). She had no history of similar eruptions. Her erythrocyte sedimentation rate was elevated $(41 \mathrm{~mm} / \mathrm{hr})$, as was her leukocyte count $\left(14100\right.$ cells $\left./ \mathrm{mm}^{3}\right)$ with marked neutrophilia (71\%). Histologic examination of a biopsy specimen obtained from her right hand showed pronounced papillary dermis edema and diffuse neutrophilic infiltration of the dermis, with no leukocytoclastic vasculitis (Figure 2). The results were negative for autoimmune and infectious diseases, as were investigations for hematological malignant disease. The results of obstetrical investigations including fetal sonography were normal. We diagnosed Sweet syndrome, and she began a 2-week course of prednisone taken orally ( $1 \mathrm{mg} / \mathrm{kg}$ each day). The cutaneous lesions and systemic symptoms resolved, and the pregnancy continued without further problems.

Sweet syndrome is a type of neutrophilic dermatoses, a group of conditions characterized by intense neutrophilic dermal infiltration without infection or vasculitis. It occurs idiopathically but may occur in association with pregnancy, drugs, infections and hematological malignant disease. ${ }^{1}$
Clinical images are chosen because they are particularly intriguing, classic or dramatic.

Submissions of clear, appropriately labelled high-resolution images must be accompanied by a figure caption and the patient's written consent for publication. A brief explanation ( 300 words maximum) of the educational significance of the images with minimal references is required.

\author{
Alexandra Maria Giovanna \\ Brunasso MD \\ Department of Dermatology \\ Prato Hospital \\ Prato, Italy \\ Cesare Massone MD \\ Research Unit of Dermatopathology \\ Department of Dermatology \\ Medical University of Graz \\ Graz, Austria
}

\section{REFERENCE}

1. Ratzinger G, Burgdorf W, Zelger BG, et al. Acute febrile neutrophilic dermatosis: a histopathologic study of 31 cases with review of literature. Am J Dermatopathol 2007;29:125-33. 